METABOLOMICS

\section{Metabolites on the inside}

Angew. Chem. Int. Ed. 51, 10386-10389 (2012)

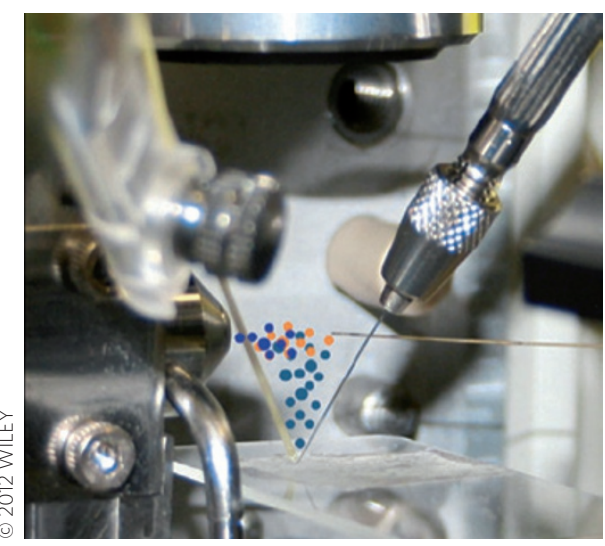

Determining the distribution and concentration gradient for a metabolite inside live cells can lead to a better understanding of its turnover, transport and compartmentalization properties. Electrospray mass spectrometry is widely used for analysing metabolites; however, most techniques for preparing a sample from a specific organelle require the organelle to be separated from the rest of the cell prior to chemical analysis, using an approach such as non-aqueous extraction. Now, Akos Vertes and co-workers at The George Washington University in the US, have combined microdissection with laser-ablation-electrospray-ionization mass spectrometry to detect metabolites localized in a subcellular compartment without the need for complicated sample preparation.

To demonstrate the potential of their method, the team analysed the metabolites inside plant cells from an Allium cepa (onion) bulb. In the first step, a microdissection needle cuts a hole in the cell membrane while leaving the organelles intact. Only a single cell is cut open during dissection and the neighbouring cells remain undamaged. In the next step, an optical fibre with a $15-\mu \mathrm{m}$ tip is used to direct a laser IR pulse at a specific region. The pulse creates a plume of ejected material that is ionized and characterized using electrospray mass spectrometry to identify the metabolites present. The sharpened tip of the optical microfibre is a similar size to the cell nucleus, which enables the team to selectively ablate it - although some cross contamination with the surrounding cytoplasm is inevitable. Aiming the optical fibre solely at the cytoplasm allowed for the selective analysis of the metabolites present outside the nucleus.

Using a combination of multi-variance statistics and a comparison of the relative intensities of the peaks, it was possible to pick out metabolites that were present in different concentrations in the cytoplasm and the nucleus. The mass spectrometry data also enabled a putative identification of the metabolites. Hexose and some secondary metabolites were found to accumulate primarily in the cytoplasm, while some amino acids are more common in the nucleus.

\section{ATMOSPHERIC CHEMISTRY}

\section{Good vibrations}

\section{Science 337, 1066-1069 (2012)}

The chief driver of chemistry in the Earth's atmosphere is the electronic excitation of molecules by light, which kick starts many important processes by photodissociating small molecules. Vibrationally excited molecules also exist in the atmosphere, but current thinking assumes that they quickly relax to equilibrium before being involved in any subsequent chemistry. Now, a team led by David Glowacki from the University of Bristol and Paul Seakins from the University of Leeds have shown that vibrationally excited molecules can play a major role in the outcome of some bimolecular reactions.

They studied the reaction of $\mathrm{OH}$ with acetylene, and the subsequent reaction with oxygen of the adduct that they form. Acetylene and $\mathrm{OH}$ react to produce a vibrationally excited hydroxyvinyl radical, of which there are two possible, and interconvertible, conformers - with the hydroxyl group taking a position either cis or trans to the nascent radical orbital. The conformers are produced in a 50:50 ratio, but on thermal relaxation this changes to 78:22 in favour of the cis form. Both adducts react with oxygen, but they give different products: the cis isomer produces ethane-1,2-dione and $\mathrm{OH}$, whereas the trans isomer reacts to give formic acid and a formyl radical. Therefore, if the onward reactions were occurring after the vibrational excitation had been quenched, as is universally assumed in such systems, the product yields would reflect the thermally equilibrated 78:22 cis:trans ratio of the adducts.

Glowacki, Seakins and colleagues measured the yield of $\mathrm{OH}$ - produced from the reaction of the cis adduct with oxygen - in an $\mathrm{O}_{2} / \mathrm{N}_{2}$ atmosphere at various temperatures, pressures and $\mathrm{O}_{2}: \mathrm{N}_{2}$ ratios. The yields that they observed showed that oxygen was reacting with the hydroxylvinyl adducts before vibrational relaxation was complete. Using a computational model to assess their results, they calculated that under atmospheric conditions, approximately $25 \%$ of the acetylene- $\mathrm{OH}$ adducts reacted while still vibrationally excited.

Written by Gavin Armstrong, Russell Johnson and Anne Pichon.

\section{blogroll}

\section{Teaching the teacher}

Pity the poor teaching assistant, and a history of chemical notation.

Being a teaching assistant (TA) is as much a learning experience as a teaching experience, a fact most apparent in the student evaluations that TAs are handed at the end of the semester. These evaluations put the spotlight on those who have been accustomed to putting it on others. On his blog, Chembark (http://go.nature.com/WrvJCs) has a whole two-courses worth of student evaluations that convey his lesson that "teaching is excellent at identifying even the smallest gaps in your knowledge, as students are quite proficient at exposing inconsistencies in the material presented to them."

Meanwhile, Master Organic Chemistry (http://go.nature.com/QaAlHX) implores undergraduates to know thy TA and realize that the TA is essentially a poor graduate student whose main allegiance is to his or her research.

And speaking of the basic chemical principles that TAs try to drill into their students' heads, do you know where those much feared and respected curly arrows came from? Henry Rzepa (http:// go.nature.com/xADRsc) traces their origins to a 1924 paper by the famous Robert Robinson. Rzepa analyses Robinson's curly arrows and finds a few deficiencies in them that diligent undergraduates should be able to point out to their TAs

Finally, Rzepa provides us with another window into chemical history (http:// go.nature.com/b4ViAL) by looking for the first noted instances of the ubiquitous wedges and dashed lines that indicate stereochemistry. After examining the work of two masters of the art Derek Barton and R. B. Woodward - a commenter points us to a fascinating collection of articles on chemical history by William Jensen that locates the first wedges and dashes in a 1932 paper by R. Kuhn.

Written by Ashutosh Jogalekar, who blogs at http://wavefunction.fieldofscience.com 